\title{
Current Utility of Chimeric Antigen Receptor T-Cell Therapy in Non-Small Cell Lung Cancer
}

\author{
Mark Jaradeh, Brett Curran and Wickii T Vigneswaran* \\ Department of Thoracic and Cardiovascular Surgery, Loyola University Medical Center, Maywood, IL, USA
}

${ }^{*}$ Corresponding author: Wickii T Vigneswaran, Department of Thoracic and Cardiovascular Surgery, Loyola University Health System 2160 S First Avenue, Maywood, IL, USA; Tel: 708327 2449; Email: wickii.vigneswaran@lumc.edu

Received: November 06, 2020; Accepted: November 16, 2020; Published: November 28, 2020

\begin{abstract}
Although the utilization of chimeric antigen receptor (CAR) T-cells for the treatment of non-small cell lung cancer (NSCLC) has traditionally been severely limited, numerous recent technological advancements have allowed for rapid progression of the field in various forms. With the maturation of techniques such as genotyping, immunohistochemistry, large-scale antibody production, and ultra-high throughput screening among many others, the production of novel NSCLC-focused CAR T-cells encompassing a wide array of structural designs and functions has yet to undergo a transition comparable to that of the previous decade. Indeed, the number and quality of modern antigens, antibodies, short-chain variable fragment (scFv) sequences, ligands, and inhibitors available for designing and bioengineering CARs have allowed for a markedly increased understanding of the mechanisms and processes necessary for the successful production of a CAR T-cell line. Most notably, advances in antigen understanding, targeting, and manipulation, CAR module integration, interaction, and compatibility, and immune cell modulation are three approaches currently at the focal point of NSCLC-focused CAR T-cell production. Herein, we briefly discuss the current status of each of these three strategies; novel targeting of NSCLC tumorspecific antigens, bispecific and physiological CAR T-cells, and inhibitory CAR T-cells, in the ongoing development of viable NSCLC management options.
\end{abstract}

Keywords: Non-small cell lung cancer, Chimeric antigen receptor, T-cell, Short-chain, Variable fragments, Antigen specificity [200]

\section{Introduction}

Despite the numerous modern-day treatments, therapies, and procedures, lung cancer continues to claim more lives than any other cancer, accounting for $23 \%$ (72,500/year) and 22\% (63,220/year) of all cancer deaths in males and females, respectively, in the United States [1]. Additionally, while other forms of cancer, such as Ewing tumor, transformed from a $0 \%$ to a $90 \% 5$-year survival rate between 1970 and 1994, lung cancer, from 1973-2000, only saw a $10.7 \%$ to $17.0 \%$ increase in 5 -year survival rate despite the addition of several modalities of treatment to the physician's arsenal [2-4]. Indeed, lung cancer, in particular non-small cell lung cancer (NSCLC), which comprises $85 \%$ of all lung cancer cases, has long eluded therapeutic interventions largely due to the lack of identified and targetable tumorspecific motifs that allow for sparing of host tissue from simultaneous destruction in addition to adequate tumor stroma penetration, solid tumor T-cell infiltration, and generation of an immune response capable of overcoming the tumor's immunosuppressive microenvironment [5].

With the advancement of immunotherapeutic techniques and approaches, however, NSCLC treatment began to dramatically evolve and, from 2000-2014, 5-year survival rate had increased from $17.0 \%$ to $21.2 \%$, or at a rate that is $29 \%$ faster than that generated through progress between 1973 and 2000 [2-4]. These new-age immunotherapeutic techniques and approaches, namely adoptive cell therapy (ACT, mainly referring to chimeric antigen receptor T-cell therapy, CAR T-cells), general/nonspecific immunotherapeutic approaches (e.g complement system-targeted approaches), monoclonal antibodies, oncolytic cancer viruses, and cancer vaccines, have all made significant progress since their introduction with CAR T-cells of the ACT subtype recently making very significant advancements for the first time in solid tumor therapy since they were first developed by Kuwana et al. in 1987 [6].

Indeed, although CAR T-cells were first produced in 1987 by Kuwana et al., it was not until 2013 that Feng et al. conducted the first clinical trial to study the safety and possibility of using CAR T-cells as immunotherapy for patients with NSCLC in which 2 out of 11 patients displayed partial response (PR) and 5 out of 11 patients had stable diseases (SD) [7]. Feng et al. used an endothelial growth factor receptor-binding (EGFR) single chain fragment variable (scFV)

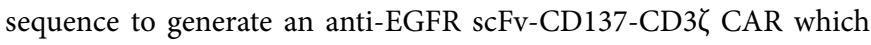
was then cloned into the lentiviral backbone pWPT which produced a plasmid that was subsequently transfected into patient CAR T cells [7]. In addition to the aforementioned results, this study also paved the way for establishing acceptable safety and toxicity outcomes of CAR T-cell therapy in that the most common adverse reactions were grades 1-2 skin irritation, nausea, vomiting, dyspnea, serum amylase elevation, and hypotension with one patient experiencing a cytokine level fluctuation-independent transient grade 3-4 serum lipase elevation [7]. 
Since Feng et al. conducted their study, numerous other groups have taken on to not only developing their own CAR T-cells, but also to modifying the molecular components of the CAR such that the cells demonstrate higher potency while simultaneously inducing fewer and less severe toxic effects [8]. In this review, we aim to summarize the current status of CAR T-cell immunotherapy and its modified derivative approaches with respect to NSCLC treatment. Although the use of CAR T-cells has yet to mature into a first line NSCLC treatment, recent developments have greatly increased the potential to effectively implement CAR T-cells in the targeting of tumor antigens and subsequent cytotoxic tumor eradication. Following is a description of newly developed CAR T-cell approaches and modifications along with a curation of newly identified, NSCLC-specific target antigens and their adoption into CAR T-cells.

\section{Novel Targeting of NSCLC Tumor-Specific Antigens}

Selectively targeting a tumor-specific antigen is one of the largest hurdles CAR T-cell therapy must overcome in order to effectively causes solid tumor regression, such as is the case in NSCLC, thus, until the recent surge in both antigen identification and antigenspecific targeting molecular candidates, development of CAR T-cells for the use in NSCLC had been largely stagnant [9-11]. For example, K1, the first monoclonal antibody isolated with affinity for mesothelin, was isolated in 1992, however, the potential to utilize it as an immunotherapeutic or diagnostic tool did not present until 2007 when Ho et al. both quantitatively and qualitatively characterized its expression in both healthy and NSCLC tissue through a combination of reverse transcription-polymerase chain reaction (RT-PCR), immunoblotting, immunohistochemistry, and flow cytometry [1214]. In characterizing its expression, Ho et al. determined mesothelin to be a therapeutic target candidate as its expression was significantly elevated in NSCLC with the mesothelin precursor protein presenting in $82 \%$ of lung adenocarcinomas and the mature form in $55 \%$ [13].

These advancements catalyzed the field's understanding of mesothelin expression as further studies, such as Kachala et al.s, found an association between mesothelin expression and reduced overall survival (OS) and recurrence free survival (RFS) rates, indicating a significant potential for mesothelin to be targeted by a CAR T-cell [15]. Multivariate analysis following adjustment for previously identified risk factors revealed an association between mesothelin expression and both reduced OS and RFS (HR $=1.78 ; 95 \% \mathrm{CI}, 1.26-2.50$; $\mathrm{P}<$ 0.01 and $\mathrm{HR}=1.67 ; 95 \% \mathrm{CI}, 1.21-2.27 ; \mathrm{P}<0.01$, respectively) which presented in vitro in the form of increased cell proliferation, invasion, and migration [15]. Furthermore, their cohort study $(\mathrm{n}=1,209)$ analyzing tissue microarrays of tumors and normal lung tissue found mesothelin expression in $69 \%$ of lung adenocarcinomas with $20 \%$ of patients expressing high levels while normal lung tissue showed no mesothelin expression, thus further implicating mesothelin as a CAR T-cell target with potential for reduced off-target toxicity [15].

In a similar manner, the membranous-bound prostate stem cell antigen (PSCA) and mucin-1 (MUC1) proteins were also found to be associated with NSCLC through protein expression studies. In the case of PSCA, Kawaguchi et al. investigated its expression in NSCLC through the analysis of primary tumors $(n=97)$ and metastatic lymph nodes $(\mathrm{n}=21)$ using immunohistochemistry and found elevated PSCA expression in 94 out of 97 primary tumors and in all metastatic lymph nodes [16]. In addition, Kawaguchi et al. found a positive correlation between PSCA expression level and advanced pathological T-factor and stage (T1 vs. T2-T4, P = 0.014 ; Stage 1 vs. Stage II-IV, $\mathrm{P}=0.029$ ) along with a significantly higher disease-free survival (DFS) rate for patients with low PSCA expression, overall insinuating a potentially pathological function of PSCA in NSCLC and its viability as a CAR T-cell target [16]. Situ et al. conducted a similar study with MUC1 through the analysis of 178 NSCLC specimens via immunohistochemistry and found elevated MUC1 expression, as defined via immunohistochemical scoring and subsequent receiver operating characteristic curve analysis, in $74.1 \%$ of NSCLCs along with associated worse OS and DFS ( $\mathrm{P}=0.011$ and $\mathrm{P}$ $=0.008$, respectively) [17]. Through multivariate analysis, MUC1 was confirmed as an independent prognostic factor for NSCLC in terms of both OS and DFS ( $\mathrm{P}=0.008$ and $\mathrm{P}=0.004$, respectively), further suggesting MUC1's role as an adverse indicator of NSCLC and thus as a potential target antigen [17].

Less than a decade later, Wei et al. investigated the significance of MUC1 and PSCA's elevated levels in NSCLC and made second generation MUC1-specific CAR T-cells and PSCA-specific CAR T-cells consisting of short-chain variable fragments $(\mathrm{scFv})$ derived from humanized 1G8 anti-PSCA and anti-MUC1 HFMG2 antibodies coupled with signaling domains from $\mathrm{CD} 28$ and $\mathrm{CD} 3 \zeta$ [18]. Lentiviral vectors encoding the CARs were transfected into pre-activated human $\mathrm{T}$ cells and final expression of anti-MUC1 and anti-PSCA CAR in $\mathrm{T}$ cells was confirmed via RTPCR analysis of the scFv sequences. Preliminary in vitro data showed significant killing of both PSCA+ and MUC1+ cell lines and confirmed PSCA-CAR and MUC1-CAR T cell specificity [18]. In vivo data generated using a PDX mouse model originating from a PSCA+, MUC1- patient tumor demonstrated significant suppression of NSCLC tumor mass growth following PRCA-CAR T-cell therapy and no significant effect in mice treated with MUC1-CAR T cells alone [18]. When MUC1 and PSCA-CAR T-cells were used to treat a PDX mouse model generated from a PSCA+, MUC1+ NSCLC patient tumor, both treatments resulted in dramatically inhibited tumor growth [18]. Furthermore, when both MUC1 and PSCA-CAR T-cells were co-administered, tumor inhibition, in the form of mass, was reduced significantly more than either MUC1 or PSCA-CAR T-cell treatment $(\mathrm{P}=0.001$ and $\mathrm{P}=0.01$, respectively) [18].

Vascular endothelial growth factor (VEGF), an angiogenic factor, has also been identified as a potential CAR T-cell target and underwent initial investigation based on the successful application of platinumbased chemotherapeutics in combination with a VEGF-A-specific $\mathrm{mAb}$ in providing an overall survival benefit for advanced disease NSCLC patients $[19,20]$. In their retrospective study, Bonnesen et al. conducted immunohistochemical studies on 102 NSCLC patient tissue samples by incubating the tissues in monoclonal antibodies to both VEGF-A and its receptor, vascular endothelial growth factor receptor 2 (VEGFR2), and assessed semi-quantitatively via intensitypercentage estimation and through Kaplan-Meier survival curves for evaluation of the proteins' expression-prognosis relationship [20]. 
Analysis showed 98 out of 102 samples expressing VEGF-A and 95 out of 102 samples expressing VEGFR2 with overall indication for poor prognosis in co-expression as shown by Seto et al. and Koukourakis et al but not according to Bonnesen et al.s analysis [20-22].

Throughout their studies, Chinnasamy et al. utilized the ubiquitous appearance of VEGFR2 in tumor vasculature and to develop a VEGFR2-CAR T-cell line that showed the ability to produce CAR T cells with not only the capacity to traffic to solid tumors, but also to operate in concert with exogenous interleukin 2 (IL-2) to enhance the immune system's ability to overcome the immunosuppression caused by the tumor microenvironment (TME) [23]. Building off of these results, Zhang et al. devised a method to engineer a CAR T-cell with inducible protein expression via IL-12 composite promoter-containing binding motif mediated through a TCR-activated nuclear factor [24]. IL-12 was chosen due to its ability to act as a proinflammatory cytokine that mediates both adaptive and innate immune responses [25]. In a subsequent study, the group tested their VEGFR2-CAR T-cells on a five different solid tumors and found that, when the tumors expressed VEGFR2, only those treated with the IL-12-producing VEGFR2-CAR T-cells were effective in mediating tumor regression and could do so without the need for any exogenous IL-2 administration as previously required [25].

Zhang et al. also focused on growth factors, however, they instead investigated EGFR variant III (EGFRvIII), a tumor-specific, mutated version of EGFR which was first documented to greatly enhance tumorigenic capacity by Nishikawa et al. in 1994 [26,27]. Zhang et al. utilized a third-generation CAR designed by subcloning EGFRvIII single chain antibody, CD8a hinge, CD28 and 4-1BB costimulatory molecules, and $\mathrm{CD} 3 \zeta$ glycoprotein into a pMSCV plasmid and subsequent transfection of the virus-packaging cell line [27]. Antitumor activity of the EGFRvIII-CART T-cell line was evaluated in vitro which revealed EGFRvIII-CAR T-cells co-cultured with EGFRvIIIexpressing A549 cells proliferate at a much higher rate than the control group, suggesting a greater ability of the cell line to express and secrete its cytotoxic factors such as perforin, granzyme B, IFN $\gamma$, and TNFa [27]. Subsequent in vivo testing in a human A549 metastatic mouse model of lung cancer revealed that, 90 days following treatment, EGFRvIII-CAR T-cell treatment significantly reduced the number of metastatic lesions formed and increased the OS to $62.5 \%$ from $0 \%$ as observed in the control group [27].

\section{Bispecific and Physiological Chimeric Antigen Receptor T-cells}

While advancements in antigen identification and modulation allowed for multiple expressed proteins to become CAR T-cell targets in the treatment of NSCLC, the risk of on-target toxicity persists as the aforementioned tumor-associated antigens (TAA) are rarely completely exclusive to malignant tissue and can frequently be found in lower numbers as a part of normal tissue. For example, with the advent of second generation CAR T-cells came an increased potency, and thus, even antigens expressed at low levels outside of the tumor were present in sufficient levels to cause an autoimmune-induced on-target toxic effect in the form of a cytokine storm such as was the case in a patient undergoing anti-ERBB2 CAR T-cell therapy for metastatic colon cancer [28]. In order to mitigate the potential for on-target toxicity, some groups, such as Lanitis et al., have turned to engineering bispecific tandem CAR T-cells in which the activating CAR component is dissociated from the costimulatory signal CAR component with the intention of requiring both undergoing independent stimulation reactions prior to any cytotoxic effect from the CAR T-cell occurring [29]. The concept behind this approach is such that the two antigens required to stimulate both the activating component and costimulatory component can be selected to both primarily reside on tumor tissue and as two targeted tumor antigens are an exceedingly rare occurrence on normal tissue, requiring the same CAR T-cell to interact with both dramatically relieves the on-target toxic burden [29]. Lanitis et al. developed one of the first bispecific tandem CAR T-cell line, opting to target mesothelin and a-folate receptor (FRa) as the group had previously constructed applicable lentiviral vector backbone constructs [30]. The antimesothelin CAR was composed of a P4 scFv linked to a CD8a hinge with transmembrane domain and connected solely via $\mathrm{CD} 3 \zeta$ signaling component while the costimulatory anti-FRa CAR was composed of the MOv-19 scFv, a CD8 $a$ hinge, and a CD28 transmembrane region and intracellular motif [30]. In vivo mouse studies demonstrated significantly more potent inhibition of tumor growth in the bispecific tandem CAR T-cell treatment group than anti-mesothelin CAR alone on tumors that coexpressed the two TAAs of interest $(\mathrm{P}=0.028)$ while simultaneously displaying much lower activity against cells displaying only one TAA of interest $(\mathrm{P}=0.0045)$ [30].

Kloss et al. employed a similar approach using prostate-specific membrane antigen (PSMA) and PSCA and encountered similar phenomena as Lanitis et al.; treatment with bispecific cells in tissues expressing both TAAs of interest resulted in significantly more potent inhibition of tumor growth than single TAA-targeting CAR T-cells $(\mathrm{P}=0.01)$, however, their anti-PSMA and anti-PSCA bispecific CAR T-cell did not spare tissues expressing single TAAs of interest [31]. This was attributed to utilizing two highly efficient CARs, thus, upon switching to a less specific scFv for PSCA, Lz1, Kloss et al. demonstrated simultaneous eradication of tumors coexpressing both TAAs and sparing of cells expressing a single TAA of interest $(\mathrm{P}=0.05$ and $\mathrm{P}=0.05)$ [31].

One of the first groups to apply bi-specific physiologic CAR T-cells to NSCLC, Chu et al., did so by developing an anti-fluorescein5-isothiocyanate (FITC) CAR to indirectly target FRa and FRb through the direct targeting of a bispecific ligand composed of FITC bound to folate to function as a bridge between the anti-FITC $\mathrm{CAR}$ and $\mathrm{FR} / \beta$, acting as a "switch" that induces the formation of a pseudoimmunological synapse [8]. Chu et al. tested the efficacy of their anti-FITC CAR T-cells in combination with folate-FITC ligand to determine whether it can redirect the anti-FITC CAR T cells to an FRa-expression A549 cell line and, through the measurement of lactate dehydrogenase $(\mathrm{LDH})$ released into culture media, determined a highly potent, cytolytic reaction had taken place $(\mathrm{EC50}=0.094$ +- $0.116 \mathrm{nM}$ ) against the A549-FRa cells while the same cells in the presence of control CAR T-cells failed to present any signs of cytolytic activity [8]. Additionally, Che et al's bispecific ligand also showed a dose-titratable, highly potent cytolytic activity towards FR $\beta$-positive cells, thus suggesting that a single CAR T-cell can not only target tumor 
cells, but also the FR $\beta$-expression tumor-associated macrophages in NSCLC [8].

\section{Next Generation CAR T-Cells: Inhibitory Chimeric Antigen Receptors}

A younger modality of CAR T-cell modification and effect modulation revolves around altering the endogenous T-cell inhibitory pathways in order to reduce potential CAR toxicity or broaden cell applicability and enhance anti-tumor efficacy [32]. An application of iCARs with strong prospects for the treatment of NSCLC constructed by Riese et al. in which a negative regulator of the T-cell receptor (TCR) signaling pathway was deleted with the intention of inhibiting an inhibitor to increase signaling efficiency [33]. Riese et al. focused on two highly expressed isoforms of diacylglycerol kinase (dgk), dgka and $\operatorname{dgk} \zeta$, which function to metabolize diacyl glycerol (DAG) such that downstream RAS and extracellular signal-regulated kinase (ERK) are limited in activation and reduce the stimulation of nuclear transcription factors [33,34]. The augmentation of TCR signal transduction is hypothesized to play a major role in overcoming CD8+ T-cell inhibition by the TME and potentially lead to a more robust anti-tumoral response [33]. Dgk $\zeta$ deficient mice were challenged with Listeria-ova in order to generate activated $\operatorname{dgk} \zeta$-deficient CD8+ T-Cells which were subsequently transferred to tumor-bearing mice and showed significantly reduced tumor size $(\mathrm{P}=0.05)$ and increased persistence of effector cells, however, tumors were not fully eradicated, thus indicating treatment via $\mathrm{dgk} \zeta$ knockout is not sufficient individually [33]. As a result, additional modification resulted in an anti-mesothelin CAR transduction into the activated CD8+ T-cells which demonstrated enhanced cytotoxicity in $\mathrm{dgk} \zeta$ single knock-out $\mathrm{T}$ cells [33]. These effects were profoundly increased in $\operatorname{dgk} \zeta$, dgka double knock-out (DKO), anti-mesothelin CAR transduced CD8+ T-cells $(\mathrm{P}=0.0001)$ along with augmentation of ERK signaling, CD 69 expression, FASL and TRAIL expression, and TGF $\beta$ resistance [33]. Lastly, DKO anti-mesothelin CAR T-cells were subcutaneously coinfected with mesothelin-expressing TC1 cells, a murine NSCLC, with tumors excised following a 10-day incubation period, the results of which suggested significant DKO anti-mesothelin CAR T-cell efficacy against the mesothelin-expressing TC1 NSCLC cell line [33].

\section{Conclusion}

The application of CAR T-cells for the treatment of solid tumors, in particular NSCLC, is a quickly developing paradigm and its many recent successes indicate it to be an increasingly promising field. As discussed, CAR T-cells, in a very short duration of time, have made tremendous progress in a field that merely a decade ago seemed utterly out of reach through the development and evolution of novel tumor-specific antigen targeting, bispecific and physiological CARs, and iCARs. While progress has been extraordinarily fast-paced and widespread, novel and ongoing investigations must continue not only in the form of developing CAR T-cells, but also bettering our understanding of the tumor microenvironment in NSCLC and the underlying mechanisms so as to develop survival prolonging techniques via a multi-faceted approach.

\section{References}

1. Rebecca L. Siegel, Kimberly D. Miller, Ahmedin Jemal (2020) Cancer statistics. CA: A Cancer Journal for Clinicians 70: 7-30.

2. Guido Pastore, Maria Luisa Mosso, Fabrizio Carnevale, Luca Cordero di Montezemolo, Marco Forni, et al. (2001) Survival trends of childhood cancer diagnosed during 1970-1994 in Piedmont, Italy: A report from the Childhood Cancer Registry. Med Pediatr Oncol 36: 481-488.

3. Janakiraman Subramanian, Thomas Regenbogen, Gayathri Nagaraj, Alex Lane, Siddhartha Devarakonda, et al. (2013) Review of Ongoing Clinical Trials in NonSmall-Cell Lung Cancer: A Status Report for 2012 from the ClinicalTrials.gov Web Site. Journal of Thoracic Oncology 8: 860-865. [crossref]

4. Claudia Allemani, Tomohiro Matsuda, Veronica Di Carlo, Rhea Harewood, Melissa Matz, et al. (2018) Global surveillance of trends in cancer survival 2000-14 (CONCORD-3): Analysis of individual records for 37513025 patients diagnosed with one of 18 cancers from 322 population-based registries in 71 countries. The Lancet 391: 1023-1075. [crossref]

5. Nan Chen, Xiaoyu Li, Navin Chintala K, Zachary Tano E, Prasad Adusumilli S (2018) Driving CARs on the uneven road of antigen heterogeneity in solid tumors. Curr Opin Immuno 51: 103-110. [crossref]

6. Yoshihisa Kuwana, Yoshihiro Asakura, Naoko Utsunomiya, Mamoru Nakanishi, Yohji Arata, et al. (1987) Expression of chimeric receptor composed of immunoglobulinderived V resions and T-cell receptor-derived C regions. Biochemical and Biophysical Research Communications, 149: 960-968. [crossref]

7. Kaichao Feng, Yelei Guo, Hanren Dai, Yao Wang, Xiang Li, et al. (2016) Chimeric antigen receptor-modified $\mathrm{T}$ cells for the immunotherapy of patients with EGFRexpressing advanced relapsed/refractory non-small cell lung cancer. Sci China Life Sci 59: 468-479. [crossref]

8. Wenqi Chu, Yixiong Zhou, Qi Tang, Min Wang, Yongjia Ji, et al. (2018) Bi-specific ligand-controlled chimeric antigen receptor T-cell therapy for non-small cell lung cancer. Biosci Trends 12: 298-308. [crossref]

9. Stefan Kiesgen, Leonardo Chicaybam, Navin Chintala K, Prasad Adusumilli S (2018) Chimeric Antigen Receptor (CAR) T-Cell Therapy for Thoracic Malignancies. J Thorac Oncol 13: 16-26. [crossref]

10. Masha Zeltsman, Jordan Dozier, Erin McGee, Daniel Ngai, Prasad Adusumilli S (2017) CAR T-cell therapy for lung cancer and malignant pleural mesothelioma. Transl Res 187: 1-10. [crossref]

11. Diana Darowski, Sebastian Kobold, Christian Jost, Christian Klein (2019) Combining the best of two worlds: Highly flexible chimeric antigen receptor adaptor molecules (CAR- adaptors) for the recruitment of chimeric antigen receptor T cells. MAbs 11: 621-631. [crossref]

12. Kai Chang, Ira Pastan, Mark Willingham C (1992) Isolation and characterization of a monoclonal antibody, $\mathrm{K} 1$, reactive with ovarian cancers and normal mesothelium. Int J Cancer 50: 373-381. [crossref]

13. Ho M, Bera TK, Willingham MC, Onda M, Hassan R, et al. (2007) Mesothelin Expression in Human Lung Cancer. Clinical Cancer Research 13: 1571-1575. [crossref]

14. Nelson Ordóñez G (2003) Application of Mesothelin Immunostaining in Tumor Diagnosis. The American Journal of Surgical Pathology 27: 1418-1428. [crossref]

15. Kachala SS, Bograd AJ, Villena-Vargas J, Suzuki K, Servais EL, et al. (2014) Mesothelin Overexpression Is a Marker of Tumor Aggressiveness and Is Associated with Reduced Recurrence-Free and Overall Survival in Early-Stage Lung Adenocarcinoma. Clinical Cancer Research 20: 1020-1028. [crossref]

16. Takeshi Kawaguchi, Masayuki Sho, Takashi Tojo, Ichiro Yamato, Takeo Nomi (2010) Clinical Significance of Prostate Stem Cell Antigen Expression in Non-small Cell Lung Cancer. Japanese Journal of Clinical Oncology 40: 319-326.

17. Dongrong Situ, Jian Wang, Yun Ma, Zhihua Zhu, Yi Hu, et al. (2011) Expression and prognostic relevance of MUC1 in stage IB non-small cell lung cancer. Med Oncol 28: 596-604. [crossref]

18. Xinru Wei, Yunxin Lai, Jin Li, Le Qin, Youdi Xu, et al. (2017) PSCA and MUC1 in non- small-cell lung cancer as targets of chimeric antigen receptor $\mathrm{T}$ cells. Oncoimmunology 6: 1284722. [crossref] 
19. Alan Sandler, Joan H Schiller, David H Johnson, Rober Gray, Michael Perry C, et al. (2006) Paclitaxel-Carboplatin Alone or with Bevacizumab for Non-Small-Cell Lung Cancer. N engl J med 355: 2542-2540. [crossref]

20. Barbara Bonnesen, Helle Pappot, Julie Holmstav, Birgit Guldhammer Skov (2009) Vascular endothelial growth factor A and vascular endothelial growth factor receptor 2 expression in non-small cell lung cancer patients: Relation to prognosis. Lung Cancer 66: 314-318. [crossref]

21. Takashi Seto, Masahiko Higashiyama, Hiroko Funai, Fumio Imamura, Kazutsugu Uematsu, et al. (2006) Prognostic value of expression of vascular endothelial growth factor and its flt-1 and KDR receptors in stage I non-small-cell lung cancer. Lung Cancer 53: 91-96. [crossref]

22. Michael I Koukourakis, Alexandra Giatromanolaki, Philip E Thorpe, Rolf A Brekken, Efthimios Sivridis, et al. (2000) Vascular Endothelial Growth Factor/KDR Activated Microvessel Density versus CD31 Standard Microvessel Density in Non-Small Cell Lung Cancer. Tumour Bilogy 60.

23. Dhanalakshmi Chinnasamy, Zhiya Yu, Marc Theoret R, Yangbing Zhao, Rajeev Shrimali K, et al. (2010) Gene therapy using genetically modified lymphocytes targeting VEGFR-2 inhibits the growth of vascularized syngenic tumors in mice. $J$ Clin Invest 120: 3953-3968. [crossref]

24. Ling Zhang, Sid Kerkar P, Zhiya Yu, Zhili Zheng, Shicheng Yang, et al. (2011) Improving Adoptive T Cell Therapy by Targeting and Controlling IL-12 Expression to the Tumor Environment. Molecular Therapy 19: 751-759. [crossref]

25. Chinnasamy D, Yu Z, Kerkar SP, Zhang L, Morgan RA, et al. (2012) Local Delivery of Interleukin-12 Using T Cells Targeting VEGF Receptor-2 Eradicates Multiple Vascularized Tumors in Mice. Clinical Cancer Research 18: 1672-1683. [crossref]

26. Nishikawa R, Ji XD, Harmon RC, Lazar CS, Gill GN, et al. (1994) A mutant epidermal growth factor receptor common in human glioma confers enhanced tumorigenicity. Proceedings of the National Academy of Sciences 91: 7727-7731.
27. Zhao Zhang, Jun Jiang, Xiaodong Wu, Mengyao Zhang, Dan Luo, et al. (2019) Chimeric antigen receptor $\mathrm{T}$ cell targeting EGFRvIII for metastatic lung cancer therapy. Front Med 13: 57-68, February. [crossref]

28. Richard Morgan A, James Yang C, Mio Kitano, Mark Dudley E, Carolyn Laurencot M, et al. (2010) Case Report of a Serious Adverse Event Following the Administration of T Cells Transduced With a Chimeric Antigen Receptor Recognizing ERBB2. Molecular Therapy 18: 843-851. [crossref]

29. Eugene A Zhukovsky, Richard J Morse, Marcela V Maus (2016) Bispecific antibodies and CARs: Generalized immunotherapeutics harnessing T cell redirection. Current Opinion in Immunology 40: 24-35. [crossref]

30. Lanitis E, Poussin M, Klattenhoff AW, Song D, Sandaltzopoulos R, et al. (2013) Chimeric Antigen Receptor T Cells with Dissociated Signaling Domains Exhibit Focused Antitumor Activity with Reduced Potential for Toxicity In Vivo. Cancer Immunology Research 1: 43-53. [crossref]

31. Christopher Kloss C, Maud Condomines, Marc Cartellieri, Michael Bachmann, Michel Sadelain (2013) Combinatorial antigen recognition with balanced signaling promotes selective tumor eradication by engineered T cells. Nat Biotechnol 31: 71-75. [crossref]

32. Fan Zou, Lijuan Lu, Jun Liu, Baijin Xia, Wanying Zhang, et al. (2019) Engineered triple inhibitory receptor resistance improves anti-tumor CAR-T cell performance via CD56. Nat Commun 10: 4109.

33. Riese MJ, L-C Wang S, Moon EK, Joshi RK, Ranganathan A, et al. (2013) Enhanced Effector Responses in Activated CD8+ T Cells Deficient in Diacylglycerol Kinases. Cancer Research 73: 3566-3577. [crossref]

34. Daniel Ritt A, María Abreu-Blanco T, Lakshman Bindu, David Durrant E, Ming Zhou, et al. (2016) Inhibition of Ras/Raf/MEK/ERK Pathway Signaling by a StressInduced Phospho-Regulatory Circuit. Molecular Cell 64: 875-887. [crossref]

\section{Citation:}

Mark Jaradeh, Brett Curran, Wickii T Vigneswaran (2020) Current Utility of Chimeric Antigen Receptor T-Cell Therapy in Non-Small Cell Lung Cancer. Cancer Stud Ther J Volume 5(4): 1-5. 